\title{
Single Exposure Wavefront Curvature Estimation of High Harmonic Radiation by Diffraction from a Regular Array
}

\author{
James S. Feehan ${ }^{1}$, Hannah M. Watts ${ }^{2}$, Jonathan H. V. Price ${ }^{1}$, Russell S. Minns ${ }^{2}$, William S. Brocklesby ${ }^{1}$, \\ Jeremy G. Frey ${ }^{2}$ \\ ${ }^{1}$ Optoelectronics Research Centre, University of Southampton, Southampton, Hampshire, SO17 1BJ \\ ${ }^{2}$ School of Chemistry, University of Southampton, Southampton, Hampshire, SO17 1BJ \\ jsf1v07@orc.soton.ac.uk
}

\begin{abstract}
We present a novel technique for estimating the radius of curvature from a single exposure of EUV light from a high harmonic source diffracted by a grating of square apertures. OCIS codes: (140.3460) Lasers; (140.7240) UV, EUV, and X-Ray Lasers
\end{abstract}

\section{Introduction}

High harmonic generation (HHG) has recently seen an expanding range of applications including coherent diffractive imaging [1], and attoscience [2]. An inherently inefficient process, investigation of HHG has predominantly been focused on the development of new sources for increased flux. However, the characterisation of these sources has been limited to spectral analysis. Recent advances in characterisation have included the spatial mapping of the spectral content [3] as well as wavefront analysis [4]. Requiring multiple exposures of the light scattered from two slits of variable separation, the low usable flux which results from traditional wavefront analysis techniques necessitates long integration times. We demonstrate a new method for estimating the radius of curvature of the generated EUV through a simple comparison between simulated and experimental data, requiring a single exposure of the scattering pattern produced by a transmissive grating. The technique was applied to HHG driven by $0.6 \mathrm{~mJ}, 40 \mathrm{fs}$ pulses from a Ti:Sapphire laser, and $0.1 \mathrm{~mJ}, 300 \mathrm{fs}$ pulses at a repetition rate of $16.7 \mathrm{kHz}$ from an $\mathrm{Yb}-$ doped fibre system, where it was tested in both the near- and far-field regions of the EUV generation volume. It is expected that the technique will be combined with the spatio-spectral mapping outlined in [3], which will allow for the full spatial characterisation of the EUV from a single exposure.

\section{Method and Results}

The experimental set-up is demonstrated in Fig. 1. A Ti:Sapphire chirped-pulse amplification (CPA) system providing $600 \mathrm{~mW}$ of average power in $40 \mathrm{fs}$ pulses at a repetition rate of $1 \mathrm{kHz}$ was used. Lenses of both $400 \mathrm{~mm}$ and $500 \mathrm{~mm}$ focal length were used to focus the $790 \mathrm{~nm}$ light to an intensity of $\sim 2 \times 10^{14} \mathrm{Wcm}^{-2}$ within the Mach disk of a gas jet backed by 300 mbar of argon held under vacuum with a background pressure of $\sim 6 \times 10^{-5}$ mbar. The EUV was separated from the residual IR by using two $200 \mathrm{~nm}$ thick aluminium foils, after which grating consisting of square apertures was placed, separated from the focus by $15-60 \mathrm{~cm}$, and from an EUV CCD by $15-60 \mathrm{~cm}$. The exposure time was typically $60 \mathrm{~s}$.

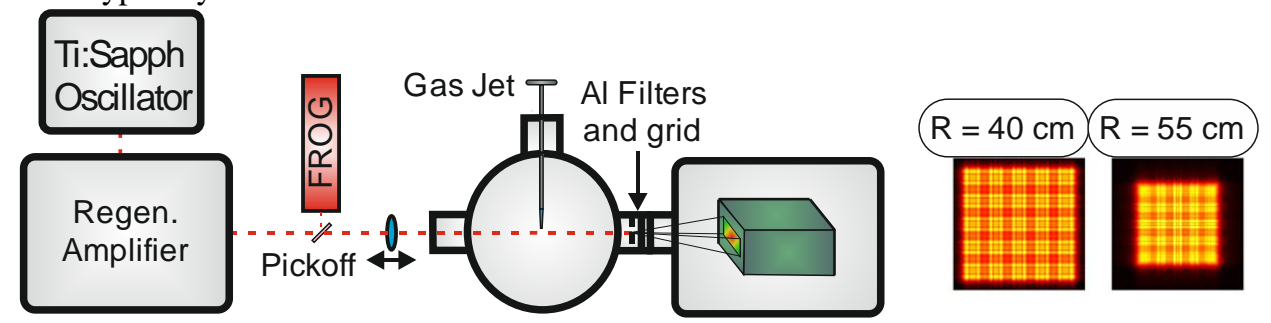

Fig. 1 Set-up for the Ti:Sapphire experiment. Inset (right): Scaling of the near-field scattering pattern with radius of curvature, $R$.

By altering the separation of the grating and the gas jet it was possible to scale the diffraction pattern. This effect became less pronounced as the grating was extended into the far-field of the focus, and has therefore been attributed to the wavefront curvature at the grating. This is demonstrated numerically in Fig. 1, in which the angular spectrum method is applied to the propagation of harmonics 19 to 27 diffracting through a grating of $300 \mu \mathrm{m}$ wide squares separated by $18 \mu \mathrm{m}$. The source, grating, and CCD separations were set to $0.4 \mathrm{~m}$ and $0.6 \mathrm{~m}$ respectively. The total diffraction pattern was calculated using the incoherent sum of those produced by the individual harmonics, the relative intensities of which were measured using a grazing incidence spectrometer. Using simulated data as the input, it was confirmed that the minima are global and unique, and are independent of grating and CCD separation. In order to test the independence of the measurements from grating type, 
two different meshes were used: one of $300 \mu \mathrm{m}$ width and $18 \mu \mathrm{m}$ separation ( $90 \%$ transmissive), the other of 200 $\mu \mathrm{m}$ width and $50 \mu \mathrm{m}$ separaion ( $63 \%$ transmissive). It was observed both numerically and experimentally that the size of the grating apertures does not alter the estimated radius of curvature, but alters the rate of convergence. This is attributed to the extent of the phase change over the aperture.

Experimentally, the radius of curvature of the EUV was extracted from the data by determining the average size of the squares in both the theoretical and experimental near-field diffraction patterns and finding the wavefront curvature of the theoretical data which minimises the error between the two. Application of the spherical wavefront curvature is supported by the literature [4]. Lenses of both $400 \mathrm{~mm}$ and $500 \mathrm{~mm}$ focal length were used, and the results are shown in Fig. 2.2. Here, the estimated radii of curvature were set to be equal $(\sim 37.8 \mathrm{~cm})$ by increasing the separation of the argon jet and grating for the longer focal length.

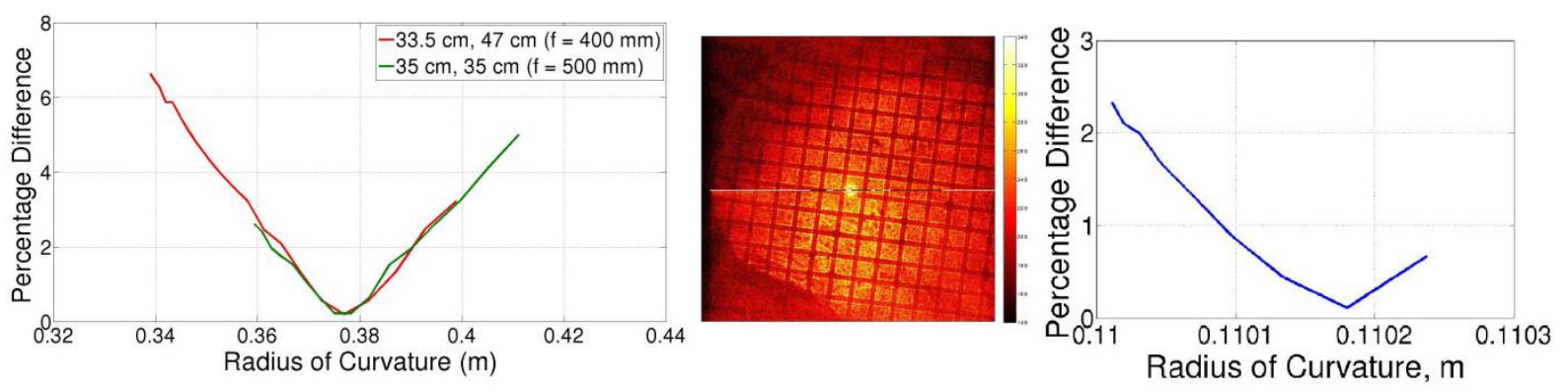

Fig. 2 Left: Experimental measurements of the radius of curvature of the EUV generated from two different focal lengths from the Ti:Sapphire system. Centre: Experimental scattering data from the Yb-fibre set-up. Right: Extracted radius of curvature for the Yb-fibre set-up.

The method was tested experimentally in the far-field of the EUV generation volume using an Yb-fibre CPA system. A focal length of $\sim 6 \mathrm{~cm}$ was used to reach an intensity of $1.5 \times 10^{14} \mathrm{Wcm}^{-2}$ within a gas cell backed with 8 mbar of xenon. The gas cell, grating, and CCD were separated by $11 \mathrm{~cm}$ and $18 \mathrm{~cm}$ respectively. As shown in Fig. 2, the radius of curvature was found to be $\sim 11 \mathrm{~cm}$, as expected from the gas cell to grating separation.

Future work will focus of merging this technique with the spatio-spectral mapping described by Praeger, et al. [3], which can be extracted from the same data set. Integration of the two techniques will allow for a full spatial characterisation of the EUV to be extracted from a single image. The technique may also be applied to monochromatic radiation, and investigation into mapping the spatial distribution and radius of curvature of each harmonic is planned.

\section{Conclusion}

We have demonstrated a novel technique for estimating the radius of curvature from diffraction data taken with a single exposure, and have shown its applicability in two very different HHG experiments. Both numerical and experimental estimations are found to be in accordance with expected results in both the near- and far-field of the EUV generation spot. Currently averaging the contribution to the curvature of the wavefront from each harmonic, the method is also applicable to monochromatic radiation, and is therefore complimentary to the spatio-spectral mapping technique.

\section{References}

[1] M. Seaberg, B. Zhang,. D. Gardner, E. Shanblatt, M. Murnane, H. Kapteyn, D. Adams, "Tabletop Nanometer Extreme Ultraviolet Imaging in an Extended Reflection Mode using Coherent Fresnel Ptychography", arXiv:1312.2049v1 [physics.optics]

[2] M. Hentschel, R. Kienberger, Ch. Spielmann, G. A. Reider, N. Milosevic, T. Brabec, P. Corkum, U. Heinzmann, M. Drescher, and F. Krausz, "Attosecond metrology,” Nature 414, 509 (2001).

[3] M. Praeger, A. M. de Paula, C. A. Froud, E. T. F. Rogers, S. L. Stebbings, W. S. Brocklesby, J. J. Baumberg, D. C. Hanna, J. G. Frey, "Spatially Resolved Soft X-Ray Spectromoetry from Single-Image Diffraction", Nature Physics 3, 176-179 (2007)

[4] D. T. Lloyd, H. O’Keefe, S. M. Hooker, "Complete Spatial Characterization of an Optical Wavefront Using a Variable-Separation Pinhole Pair", Optics Letters 38, 1173-1175 (2013) 